\title{
On the Possibility of an Isochoric Phase Transition in an Electrostatic Field
}

\author{
A. A. Sobko \\ A.M. Prokhorov Academy of Engineering Sciences, Presnensky Val Street 19, Moscow 123557, Russia
}

\begin{abstract}
In the present paper that considers the possibility for modification of equation of state for a non-polar gas in a closed thermally-insulated capacitor and, consequently, the possibility for changing the temperature and pressure under electrostatic field. This can be classified as a new type of phase transitions.
\end{abstract}

Key words: Non-polar gas, equation of state, generalized equation of Van der Waals-Berthelot, phase transition, electrostatic field.

\section{Introduction}

Let us consider a closed thermally isolated volume (rectangular prism) filled with a non-polar gas. An arbitrary non-polar gas can be regarded here. The opposite faces of the prism form a plane capacitor and a voltage can be applied to this system.

The volume of the prism $V(P, T)$ is constant, and, thus, the pressure $P$ is uniquely determined by the given temperature $T$.

When a voltage is applied to the capacitor plates, an additional term appears in the equation of state due to dipole interaction. The induced dipole moment is proportional to the electric field strength $E$, and, consequently, the energy of the dipole interaction is proportional to $E^{2}$. Alongside with that, rotation of the dipoles will be induced by the electrostatic field leading to the appearance of another additional term in the equation of state.

2. Calculation of temperature and pressure changes within the framework of the van der Waals equation

Let us write the new expression using the volume $V_{n}(P, T)$ expansion up to the second order for the

Corresponding author: Aleksandr Aleksandrovich Sobko, director, research fields: thermodynamics, statistical physics, quantum mechanics. equation of state:

$$
V_{n}(P, T)=V_{o}(P, T)+\frac{\partial V_{o}}{\partial P} \Delta P+\frac{\partial V_{o}}{\partial T} \Delta T+\ldots,
$$

where $\Delta P$ and $\Delta T$ are the changes in pressure and temperature under application of the electric field.

The volume $V(P, T)$ is constant, hence, $V_{n}(P, T)=V_{o}(P, T)$ and, consequently, the following condition is satisfied:

$\frac{\partial V_{o}}{\partial P} \Delta P+\frac{\partial V_{o}}{\partial T} \Delta T=0$

To calculate the $\left(\frac{\partial V_{o}}{\partial P}\right)_{T}$ and $\left(\frac{\partial V_{o}}{\partial T}\right)_{P}$

derivatives one of the model equations of state can be utilized. Van der Waals or Berthelot equations can be used, for example shown in Ref. [1].

The van der Waals equation for one mole of a gas has the form:

$$
\left(P+\frac{a}{V^{2}}\right)(V-b)=R T
$$

or

$$
V^{3}-\left(b+\frac{R T}{P}\right) V^{2}+\frac{a V}{P}-\frac{a b}{P}=0 .
$$

The $\left(\frac{\partial V_{o}}{\partial P}\right)_{T}$ and $\left(\frac{\partial V_{o}}{\partial T}\right)_{P}$ derivates are calculated below. The relationship between the 
changes in temperature and pressure under application of the van der Waals model as well. The calculations of the electrostatic field is obtained in the framework according to the other models are similar.

$$
\begin{aligned}
& 3 V^{2}\left(\frac{\partial V}{\partial P}\right)_{T}-2 V\left(b+\frac{R T}{P}\right)\left(\frac{\partial V}{\partial P}\right)_{T}+\frac{R T}{P^{2}} V^{2}+\frac{a}{P}\left(\frac{\partial V}{\partial P}\right)_{T}-\frac{a V}{P^{2}}+\frac{a b}{P^{2}}=0, \\
& {\left[3 V^{2}-2 V\left(b+\frac{R T}{P}\right)+\frac{a}{P}\right]\left(\frac{\partial V}{\partial P}\right)_{T}+\frac{\left(R T V^{2}-a V+a b\right)}{P^{2}}=0,} \\
& 3 V^{2}\left(\frac{\partial V}{\partial T}\right)_{P}-2 V\left(b+\frac{R T}{P}\right)\left(\frac{\partial V}{\partial T}\right)_{P}-\frac{R V^{2}}{P}+\frac{a}{P}\left(\frac{\partial V}{\partial T}\right)_{P}=0, \\
& {\left[3 V^{2}-2 V\left(b+\frac{R T}{P}\right)+\frac{a}{P}\right]\left(\frac{\partial V}{\partial T}\right)_{P}-\frac{R V^{2}}{P}=0, \frac{\left(V_{P}^{\prime}\right)_{T}}{\left(V_{T}^{\prime}\right)_{P}}=\frac{\left(a V-R T V^{2}-a b\right)}{R P V^{2}},} \\
& \left(\frac{\partial V}{\partial T}\right)_{P}=\frac{R P V^{2}}{\left(a V-R T V^{2}-a b\right)}\left(\frac{\partial V}{\partial P}\right)_{T},\left(\frac{\partial V}{\partial P}\right)_{T} \Delta P+\left(\frac{\partial V}{\partial T}\right)_{P} \Delta T=0, \\
& \left(\frac{\partial V}{\partial P}\right)_{T} \Delta P+\frac{R P V^{2}}{\left(a V-R T V^{2}-a b\right)}\left(\frac{\partial V}{\partial P}\right)_{T} \Delta T=0,\left(\frac{\partial V}{\partial P}\right)_{T} \neq 0, \Delta P=\frac{R P V^{2}}{\left(R T V^{2}-a V+a b\right)} \Delta T .
\end{aligned}
$$

To determine the volume in the following equation:

$$
\Delta P=\frac{R P V^{2}}{\left(R T V^{2}-a V+a b\right)} \Delta T .
$$

It is sufficient to solve the cubic equation:

$$
V^{3}-\left(b+\frac{R T}{P}\right) V^{2}+\frac{a V}{P}-\frac{a b}{P}=0
$$

for given values of $P$ and $T$. The problem-solving procedure is described in algebra, for example in Ref. [2]. The constants $a$ and $b$ for specific gasses are given in the literature, for example in Ref. [1].

\section{Estimation of the change in temperature when the electrostatic field is turned on}

Let us use the following considerations for the estimation of the temperature change under application of the electrostatic field:

(1) The heat required for changing the temperature of the system is $Q=\frac{3}{2} R n \Delta T$, where

$\frac{3}{2} R$ is the heat capacity of one mole of a gas, $n$ is the number of moles.

(2) The only heat source is the work done by the external field on charge separation $A=q \operatorname{sn} N_{A} E$, the charge displacement $s$ can be evaluated as $s \approx \sqrt[3]{\alpha}$, $\alpha$ is the gas polarizability. For argon $\alpha=1.65 \cdot 10^{-30} \mathrm{~m}^{3}, \mathrm{~s} \approx 1.2 \cdot 10^{-10} \mathrm{~m}$ [3].

(3) Equating the work done on charge separation to the heat required for changing the temperature we obtain:

$$
\begin{aligned}
& Q \approx A, \frac{3}{2} R n \Delta T \approx q S n N_{A} E, \Delta T \approx \frac{2 q s N_{A} E}{3 R} \approx \frac{2 q s N_{A}}{3 R} \frac{U}{d}, \\
& \Delta T \approx \frac{2 \cdot 1.6 \cdot 10^{-19} \cdot 1.2 \cdot 10^{-10} \cdot 6 \cdot 10^{23}}{3 \cdot 8.3} \frac{U}{d} \approx 10^{-6} \frac{U}{d} .
\end{aligned}
$$

As can be seen from the expression given above a several-degree change in the temperature can be obtained with the applied voltage of a several $\mathrm{kV}$ and the spacing between the capacitor plates of a several 
$\mathrm{mm}$. The charge used for the estimation of $\Delta T$ was taken to be equal to the electron elementary electric charge $e=1.6 \cdot 10^{-19} \mathrm{~K}$. Actually, the separated charges are considerably greater and the expression that should be used for the estimation of the temperature change is $\Delta T \approx 10^{-6} Z \frac{U}{d}$, where $Z$ is the nuclear charge.

\section{Conclusions}

(i) The calculations given above indicate that the changes in temperature and pressure occur under application of electrostatic field to a closed thermally isolated capacitor filled with a non-polar gas. These changes are given by:

$$
\Delta T \approx 10^{-6} Z \frac{U}{d}, \Delta P=\frac{R P V^{2}}{\left(R T V^{2}-a V+a b\right)} \Delta T .
$$

The expression for the pressure change was obtained in the framework of the van der Waals gas model. Implementation of the other models will lead to a different result for the pressure change, but the corresponding numerical values will be just slightly different.

(ii) Given the experimental support of the considered effect one can suggest the existence of a new type of phase transitions. This transition can be classified neither as the first-order phase transition with $\Delta Q \neq 0, \Delta V \neq 0$ nor as the second-order phase transition with $\Delta Q=0, \Delta V=0$. It can be referred to as the sesquialteral-order isochoric phase transition with $\Delta Q \neq 0, \Delta V=0$.

(iii) When the electrostatic field is removed, the temperature and the pressure of the gas in the thermally isolated system will be conserved at the values of $T+\Delta T$ and $P+\Delta P$, respectively. When the electrostatic field is applied again to the temperature which will rise by $\Delta T$. Therefore, heating of a gas driven by the energy of the electrostatic field in a capacitor can be achieved.

\section{References}

[1] Prigogine, I., and Kondepudi, D. 1998. Modern Thermodynamics: From Heat Engines to Dissipative Structures. West Sussex: John Wiley \& Sons Ltd.

[2] Rashevskii, P. K., ed. 1965. Advanced algebra. Moscow: Nauka Publishers.

[3] Scherachenko, L. A. 2005. Physics of Dielectrics, Methodological Rationale. Irkutsk: Irkutsk State University. 